\title{
Novel Method for Measuring Fine Particle Distribution in Shock- Induced Atomization
}

\author{
T. Yada ${ }^{1}$, K. Mizuno ${ }^{1}$, T. Kamiya ${ }^{1}$, M. Asahara ${ }^{* 2}$, T. Miyasaka ${ }^{2}$ \\ ${ }^{1}$ Graduate School of Engineering, Gifu University, Gifu, Japan \\ ${ }^{2}$ Faculty of Engineering, Gifu University, Gifu, Japan \\ *Corresponding author email : asahara@gifu-u.ac.jp
}

\begin{abstract}
The objective of this study is to obtain the particle size distribution of fine droplets formed by a breakup behind the shock wave. The algorithm for measuring the droplet diameter using the shadow sizing method was evaluated. The images of the liquid droplet breakup behavior were obtained using a high-speed camera attached to a long-range microscope. Calibration experiments were performed to obtain the relationship between the particle-camera distance, particle size, and particle image, and an algorithm was developed to derive a surface equation that identifies the particle-focus distance $(-2 \mathrm{~mm}$ to $2 \mathrm{~mm}$ ) within $0.3 \mathrm{~mm}$ error in depth of field and particle size $(5-80 \mu \mathrm{m})$ within $6 \mu \mathrm{m}$ error. The proposed particle size distribution measurement method was used to measure the particle size distribution of fine droplets by shock-induced atomization, and the volume probability density distribution of fine droplets in atomization was obtained in accordance with previous studies.
\end{abstract}

\section{Keywords}

Shadow sizing, Droplet atomization, Fine particle size, Shock wave

\section{Introduction}

In the combustor of a scramjet engine, which is expected to be a next-generation supersonic aircraft engine, liquid fuel is injected into a supersonic airstream and undergoes atomization, evaporation, mixing with air, and combustion. The size of the fine droplets formed by atomization of the liquid fuel has a significant effect on evaporation and combustion. Therefore, it is necessary to clarify the particle size distribution of the fine droplets formed by atomization by high-speed airflow. However, the range of the experimental results is too narrow to obtain a sufficient number of samples in a single experiment; thus, repeated experiments are required. Therefore, it is important to have a wide measurement range for fine droplet size measurement techniques to efficiently obtain the particle size distribution.

Laser diffraction [1], phase Doppler [2], and shadow sizing [3-5] are the main methods used to measure the size of fine droplets. The laser diffraction method and the phase Doppler method have the advantage that the particle size can be measured with high accuracy; however, the measurement range is narrow. However, the shadow sizing method has a wider measurement range, and more data can be obtained in a single experiment. Therefore, the shadow sizing method was adopted to measure the particle size distribution of shock-induced atomization.

There are many ways to obtain the particle size using the shadow sizing method, and two typical methods are introduced here. One is to binarize the image by setting the particle contour threshold value in the luminance information in the image, and the other is to define the high luminance gradient value as the particle contour. In previous studies, the particle contour with the lowest measurement error at the point of focus was derived from calibration experiments [3, 4]. However, the derivation of optimal particle contour brightness or 
measurement accuracy has not been verified at out-of-focus locations, and there is concern that large measurement errors may occur. The depth of field of a particle is important for obtaining the number density distribution, and the depth position of a particle changes with the distance from the focal point.

Therefore, the purpose of this study is to derive the optimal contour threshold for out-of-focus particles and propose a method for identifying the depth position from the luminance information in the particles. In the calibration experiment, the calibration particles were photographed at different distances from the camera to obtain three-dimensional data of particle size, particle depth position, and particle contour brightness. Using these data, a method for identifying the exact particle size and spatial coordinates of the particles will be investigated. In addition, the fine droplets formed by droplet atomization in a high-speed air flow were photographed, and their particle size distributions were obtained using the proposed method.

\section{Method of calibration experiment}

Figure 1(a) illustrates the photographic results of the fine droplets formed by droplet atomization. Focusing on particles $A, B$, and $C$ shown in Figure 1(a), the intensity of the particle shade and the sharpness of the contour are different depending on the different positions in the depth direction in the camera. Particle A, which is close to the focal point, has a darker shade and a clearer outline, while particle $\mathrm{C}$, which is far from the focal point, has a lighter shade and an indistinct outline. Using these characteristics, we investigated whether it is possible to identify the depth direction coordinates of the particles. Therefore, solid particles whose particle size can be measured are photographed with a measurement range of $0.5-10$ $\mathrm{mm}$ and a measurement interval of $0.0025-0.1 \mathrm{~mm}$, depending on the size of the particles, and a database of camera axial coordinates, particle swarm photographed images, and particle size is constructed. Figure 1(b) shows the calibration system. A backlight light source and high-speed camera were used in this experiment. A calibration particle placed on an objective stage attached to a motorized stage was photographed using a high-speed camera with a long-range microscope. A short-pulse laser was used as the backlight, which was irradiated vertically downward from the top of the device through an optical fiber and a diffusion lens. Because it is difficult to fine-tune the emission intensity of this pulsed laser, the background luminance value of the captured image can be adjusted by attaching ND filters of various optical densities to the tip of the diffusion lens. Table 1 shows the microscope magnification, lens and teleconverter, corresponding total magnification, and pixel width $h$ on the image when measuring grain size. The distance between the camera and the particle was varied by a motorized stage that moved vertically with respect to a fixed pulsed laser and highspeed camera. The diameter of a particle identical to the one photographed in the environment shown in the Figure 1(b) was measured by magnifying it with a scanning electron microscope. Because the particles used in the calibration experiment were solid and those used in the atomization experiment were liquid, there is a difference in the presence or absence of a glare point in the middle of the captured particles. However, it does not interfere with the measurement method because the particle diameter is measured from the area within the contour extraction. 


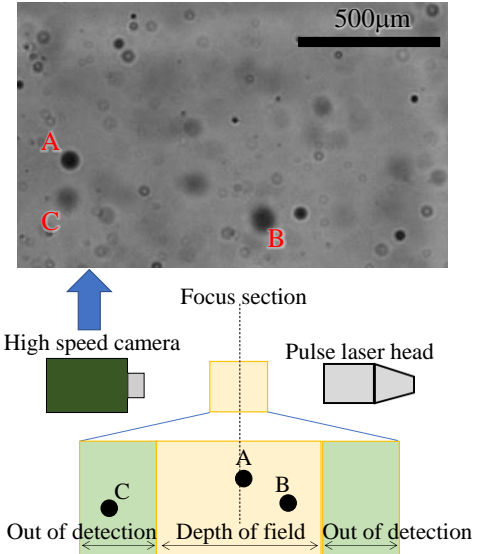

(a)

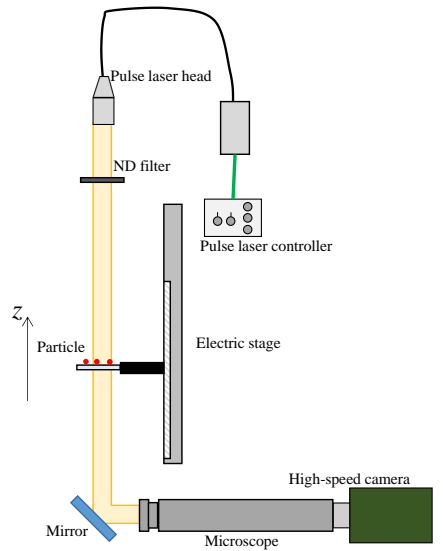

(b)

Figure 1. Calibration apparatus for particle size and number density measurement.

Table 1 - Magnification and pixel width on the picture.

\begin{tabular}{ccccc}
\hline $\begin{array}{c}\text { Microscope } \\
\text { magnification }\end{array}$ & $\begin{array}{c}\text { Lens } \\
(\times 2.5)\end{array}$ & $\begin{array}{c}\text { Teleconverter } \\
(\times 2)\end{array}$ & $\begin{array}{c}\text { Total } \\
\text { magnification }\end{array}$ & $\begin{array}{c}\text { Pixel width } h \\
{[\mu \mathrm{m}]}\end{array}$ \\
\hline$\times 3.2$ & $\bigcirc$ & $\times$ & 8 & 2.76 \\
$\times 5$ & $\bigcirc$ & $\times$ & 12.5 & 1.77 \\
$\times 9.2$ & $\bigcirc$ & $\times$ & 23 & 0.96 \\
$\times 9.2$ & $\bigcirc$ & $\bigcirc$ & 46 & 0.53 \\
\hline
\end{tabular}

\section{Method of calibration experiment}

As shown in Figure 2(a), the minimum luminance value in the particle and the depth position of the particle has a quadratic correlation (see Figure 3(a)). Figure 2(b) shows the extracted contours and the resulting intra-particle minimum luminance values when the particle contour luminance values are set. The same is true for the depth position of the particle and the particle contour threshold (see Figure 3(c)). Therefore, the approximate surface equation is

$$
z / d=\sum_{n=1}^{3} \sum_{m=1}^{3} a_{m n}(d / h)^{n} I_{\min }{ }^{m}, A=\left[a_{m n}\right]=\left[\begin{array}{ccc}
-8.74 & 4.46 & 1.89 \times 10^{2} \\
-7.14 \times 10^{-2} & -3.23 & 0 \\
7.32 & 0 & 0
\end{array}\right]
$$

which identifies the dimensionless depth position $z / d$ of a particle from the minimum luminance value within a particle normalized by the dimensionless particle size $d / h \cdot$ and the background luminance, $I_{\min }($ Figure 3(b)), and the approximate surface equation

$$
I_{t}=\sum_{n=1}^{3} \sum_{m=1}^{3} b_{m n}(d / h)^{n}(z / d)^{m}, B=\left[b_{m n}\right]=\left[\begin{array}{ccc}
6.27 \times 10^{-1} & -1.15 \times 10^{-2} & -5.43 \times 10^{-3} \\
2.36 \times 10^{-2} & 3.19 \times 10^{-4} & 0 \\
4.87 \times 10^{-5} & 0 & 0
\end{array}\right]
$$

which identifies the particle contour threshold $I_{t}$ (see Figure $3(\mathbf{b})$ ) from the dimensionless particle size $d / h$. The dimensionless depth position $z / d$ is derived by the least-squares method. Although the surface equation depends on the image capturing conditions because the calibration data required depends on the image capturing conditions, the algorithm for deriving the surface equation is the same, so it is possible to derive the surface equation for various conditions by providing the appropriate calibration data. The two derived surface equations and particle images cannot be solved algebraically. Therefore, the following procedure is used to obtain the dimensionless depth position $z / d$ and dimensionless particle diameter $d / h$ along the surface equation: 
1. Set the particle contour threshold $I_{t, n}$ to the luminance distribution near the particle in the image to define a provisional particle contour.

2. Obtain the minimum luminance value $I_{\min , n}$ and the dimensionless particle diameter $d / h_{n}$ from the luminance and shape information of the particle obtained by defining the particle contour.

3. Substituting the obtained minimum brightness value $I_{\min , n}$ and the dimensionless particle diameter $d / h_{n}$ into Equation (1) is substituted to obtain the dimensionless depth position $z / d_{n}$ of the particle.

4. Substituting the obtained dimensionless depth position $z / d_{n}$ and the dimensionless particle diameter $d / h_{n}$ obtained in step 2 into Equation (2) to obtain the optimal particle contour threshold $I_{t, n+1}$ for the loop in question.

5. Repeat steps 1-4 again using $I_{t, n+1}$ obtained in step 4, and exit the loop when $\mid d / h_{n^{-}}$ $d / h_{n-1} \mid / d / h_{n}<0.0001$ is satisfied.

To evaluate the accuracy of the method, we applied the method to calibrated particles whose particle size and particle-focus distance are both known. We define the error of the particle-focal distance

$$
z_{\text {err }}=\left|z_{\text {true }} z_{m}\right|
$$

using the particle-focal distance $z_{m}$ measured by the method, and the actual particle-focal distance $z_{\text {true }}$. The results show that the error is within $z_{e r r}<0.3 \mathrm{~mm}$ at $z=0-2 \mathrm{~mm}$ (see Figure 4(c)). In addition, the error

$$
d_{\text {err }}=\left|d_{\text {true }}-d_{m}\right|
$$

using the measured particle size $d_{m}$ and the true particle size $d_{\text {true }}$ is within $6 \mu \mathrm{m}$ at $z=0-2 \mathrm{~mm}$, indicating that the accuracy of this method is higher than that of setting it to a constant value at $z$ $=0.3-1.7 \mathrm{~mm}$ (see Figure 4(d)).
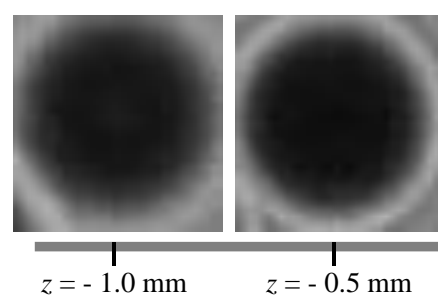

$z=-0.5 \mathrm{~mm}$

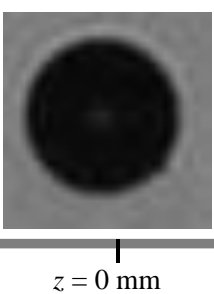

(a)

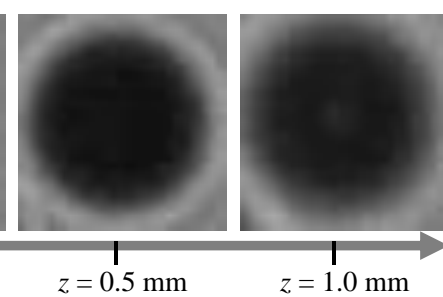

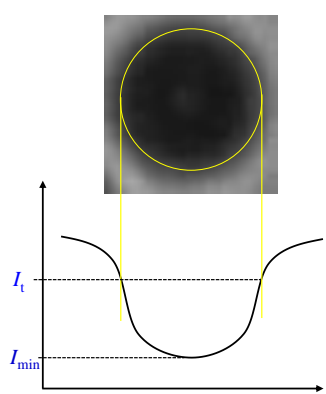

(b)

Figure 2. (a) Differences in images due to different distances from the particle focus; (b) The minimum luminance value $I_{\min }$ in the particle obtained from the particle contour extracted by setting the particle contour luminance value $I_{t}$. 


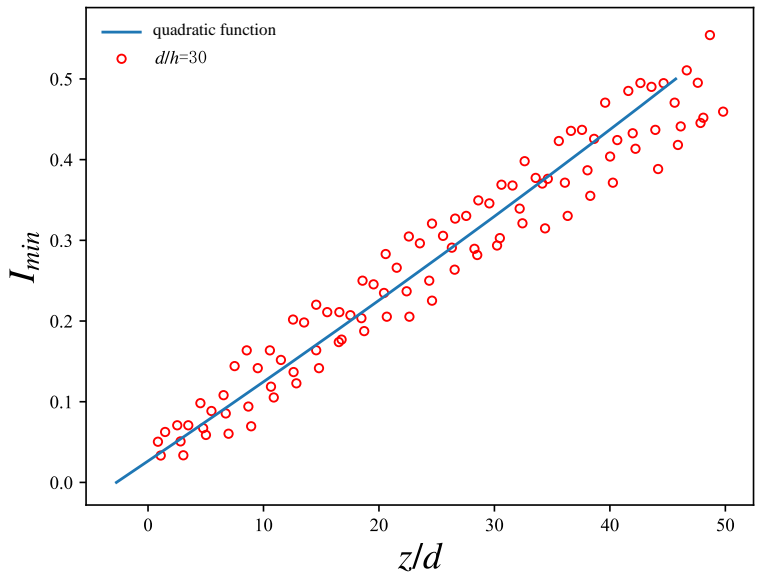

(a)

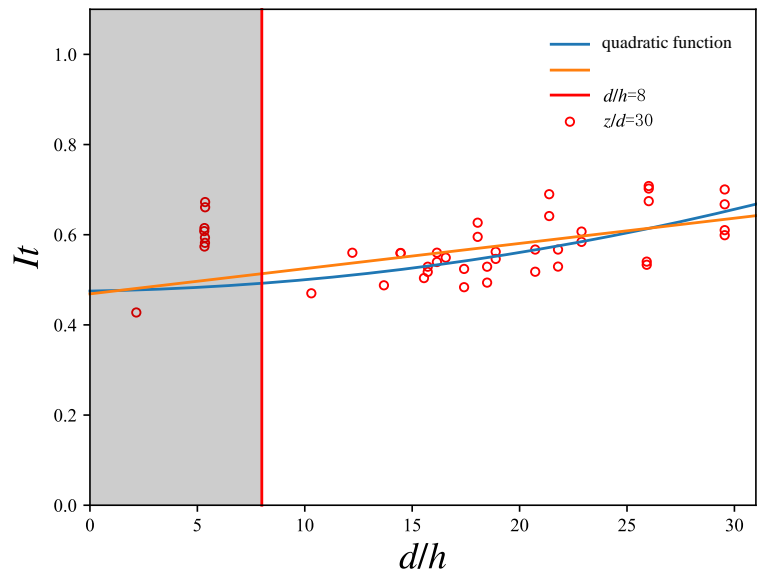

(b)

Figure 3. (a) Relationship between the depth position of a particle and the minimum luminance value in the particle at dimensionless particle size $d / h=30$; (b) Relationship between dimensionless particle size $d / h$ and particle contour threshold $I_{t}$ at dimensionless depth position $z / d=30$

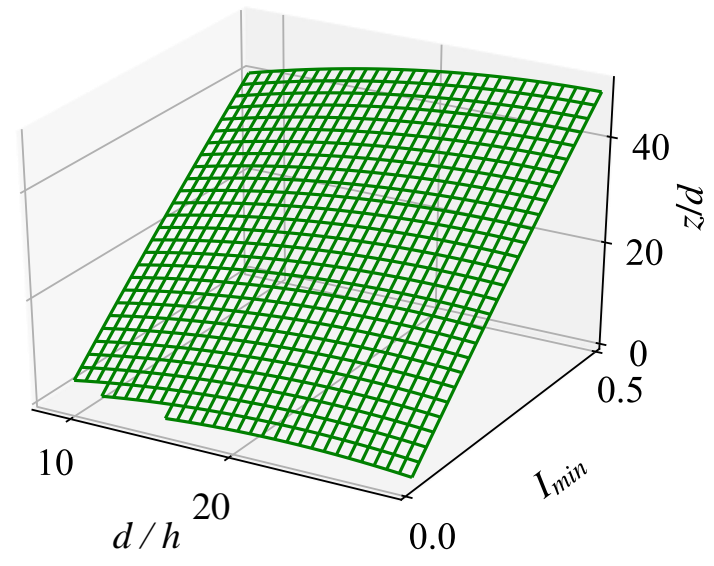

(a)

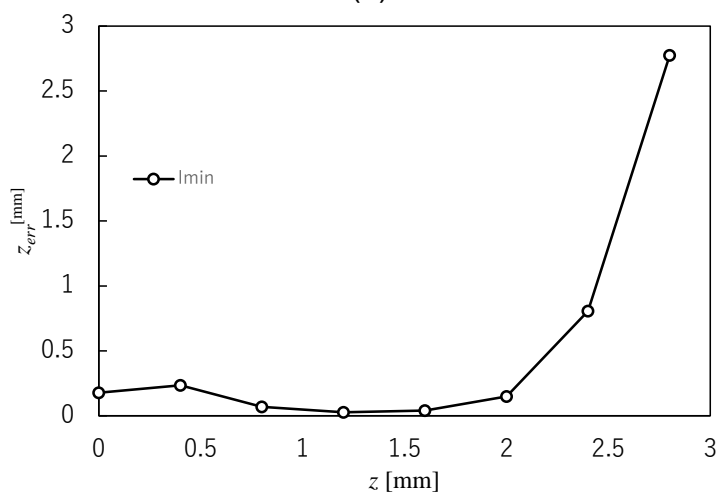

(c)

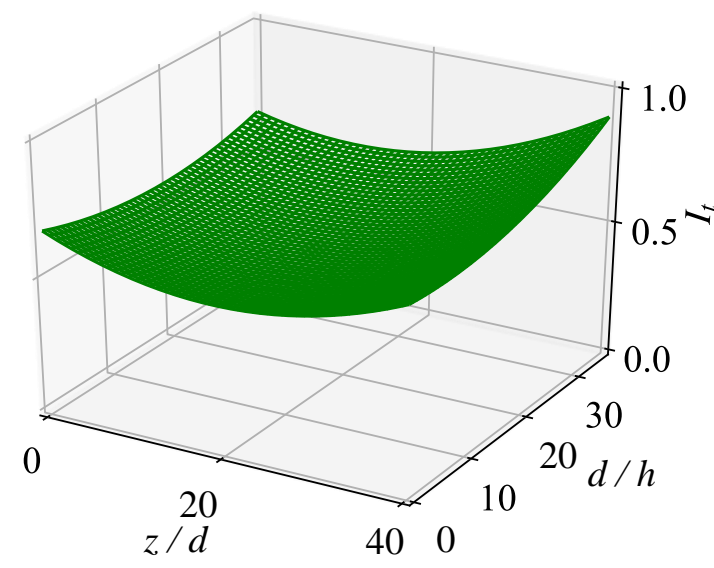

(b)

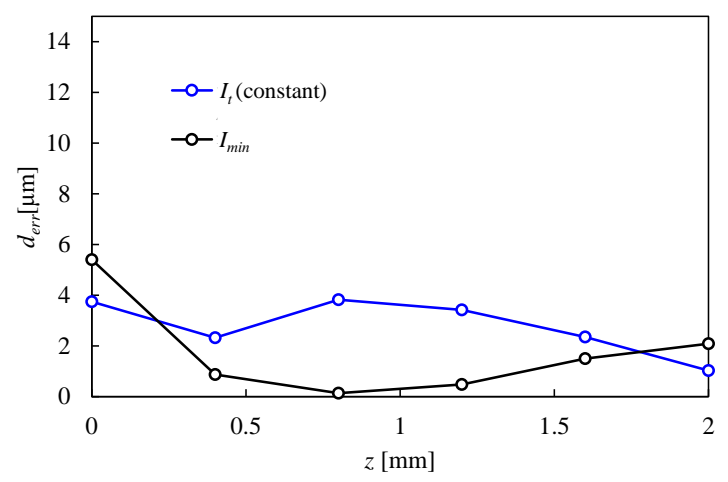

(d)

Figure 4. (a) Surface equation to identify the depth position of a particle; (b) Surface equation to identify the luminance threshold that determines the particle contour; (c) The error between the depth position $z$ of the particle derived from the surface equation and the actual depth position to the depth of field; (d) The error between the derived particle size and the true size when the particle contour threshold is set to a constant value, looped at the mean intensity value within a particle and looped at the minimum intensity value. 


\section{Atomization photography experiment}

To photograph the atomization of a single droplet, we constructed a droplet atomization observation system using a shock wave tube (see Figure 5(a)). The system uses a laser and a laser detector to detect the drop of a single droplet, which triggers the generation of a shock wave and the start of a wide-area imaging camera to locate the phenomenon. Experiments with high responsiveness and reproducibility are possible using the laser-break method [5]. A narrow-area camera was used to obtain the fine droplet diameter, and a wide-area camera was used to capture the entire atomization phenomenon, as shown in Figure 5(b). By using the laser-break method and two cameras, it is easy to obtain a large number of samples in multiple Weber number conditions.

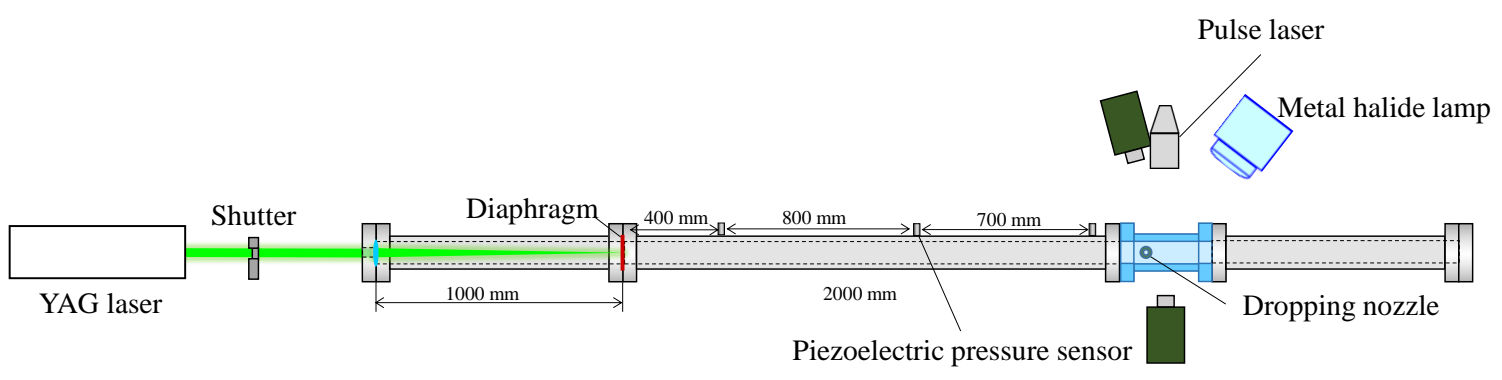

(a)

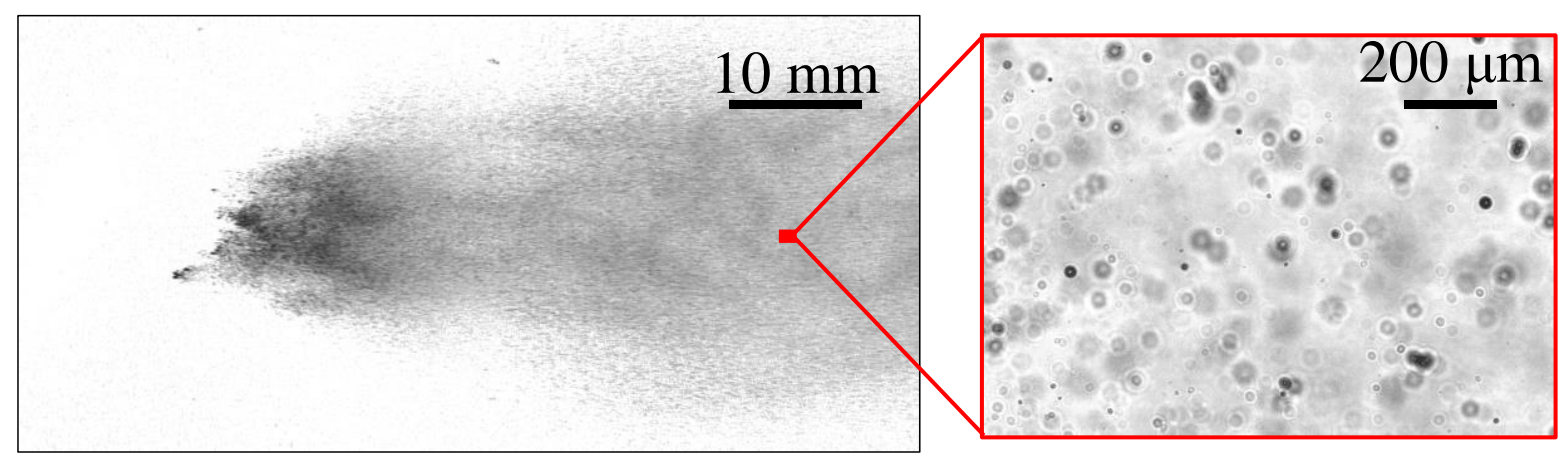

(b)

Figure 5. (a) Experimental apparatus for photographing the atomization of a single droplet; (b) Atomized image of a single droplet taken by a wide-area camera for locating the phenomenon and by a boundary area camera for measuring the particle size.

Table 1 - Physical properties of single droplet atomization experiments using shock waves.

\begin{tabular}{c|c|c|c|c|} 
No. & We & $M_{s}$ & $\operatorname{Re} \times 10^{4}$ & Sample, $N$ \\
\hline 1 & 204 & 1.12 & 1.21 & 34 \\
2 & 561 & 1.20 & 2.07 & 47 \\
3 & 1282 & 1.30 & 3.34 & 39 \\
4 & 1941 & 1.36 & 4.25 & 54
\end{tabular}




\section{Particle size distribution determination}

The time series of microdroplet images obtained from the single droplet atomization experiment is shown in Figure 6(a), with the size measurement area indicated by the red frame. The volume probability density was calculated for a group of fine droplets in a single droplet atomization with Weber number 1282 using the method for calculating the particle size and particle-focus distance shown in this study. The root-normal distribution was fitted to the volume probability density distribution (Figure 6(b), (c)). $\tau$ is the time required for a rigid sphere to move by its diameter. The root-normal distribution is represented by

$$
p(d)=\frac{1}{2 d_{\sigma r} d \sqrt{2 \pi d}} \exp \left[-\frac{\left(\sqrt{d-d_{\mu r}}\right)^{2}}{2 d_{\sigma r}{ }^{2}}\right]
$$

Guildenbecher et al. [7] stated that the volume probability density of droplet atomization is in good agreement with the root-normal distribution. The volume probability density distribution obtained by the method presented in this study shows a similar trend, and the method was considered valid.

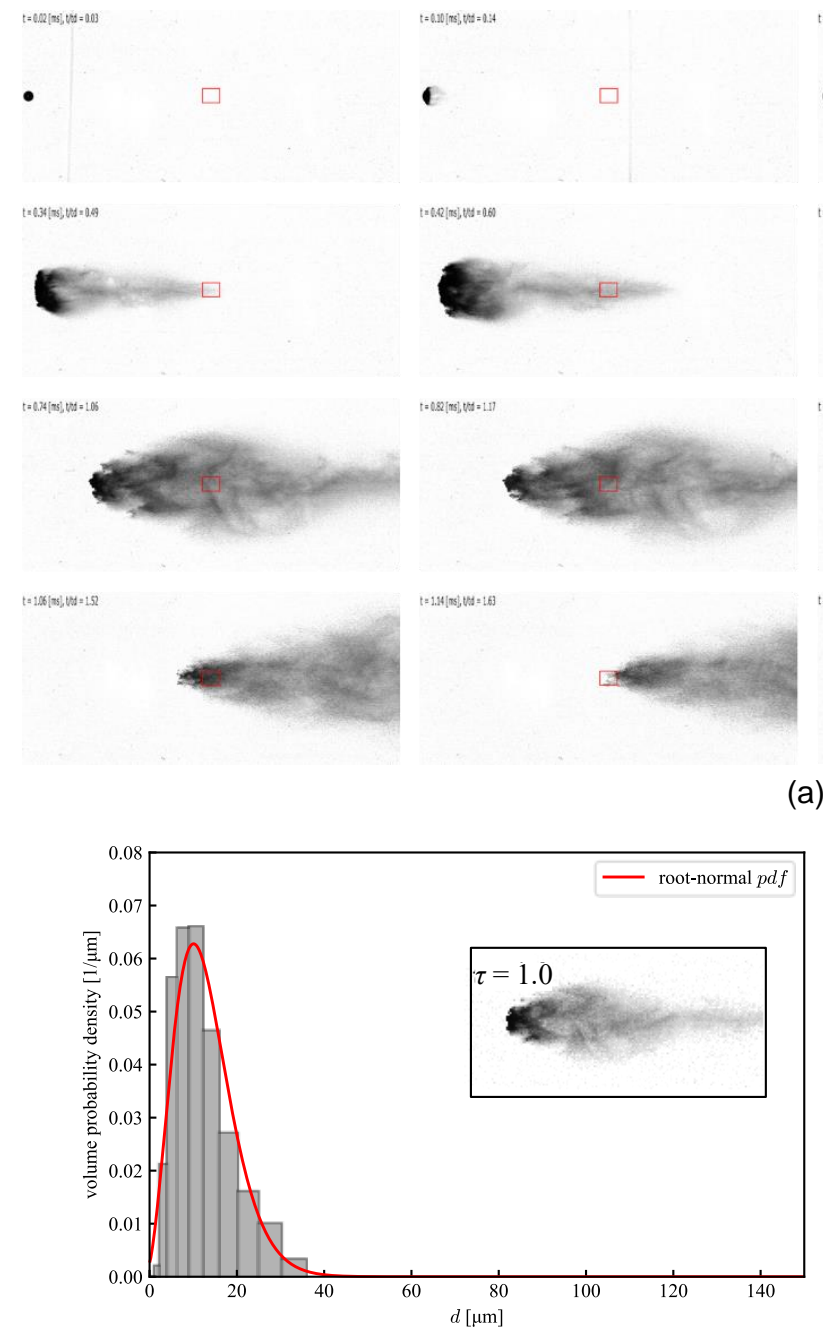

(b)
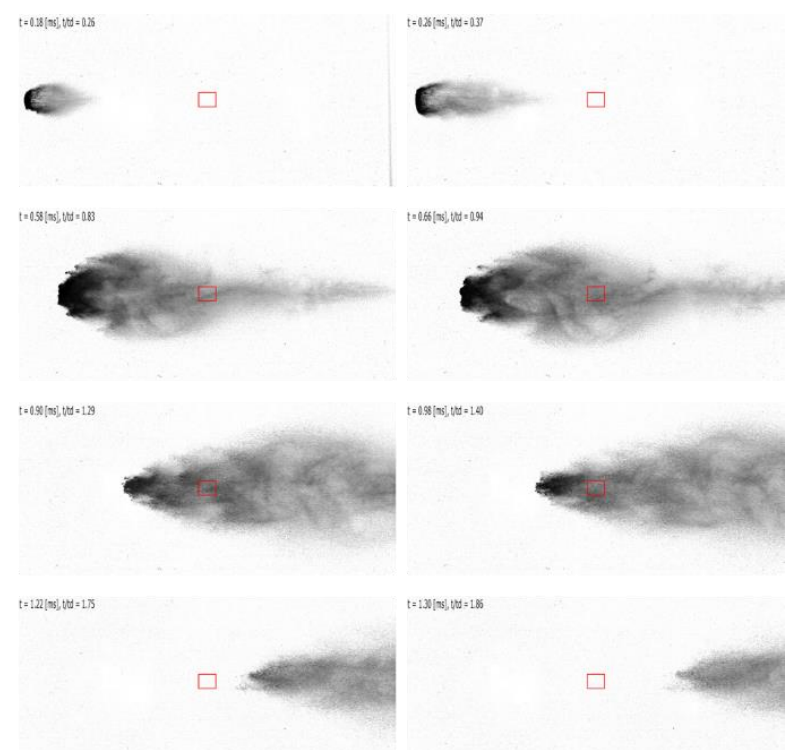

(a)

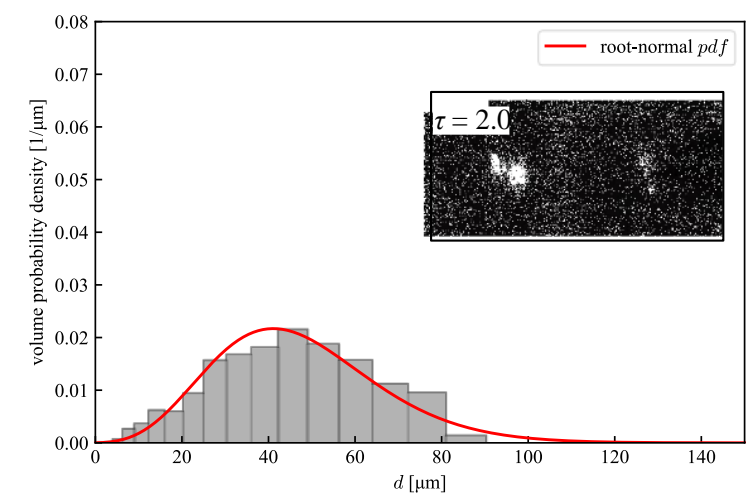

(c)

Figure 6. (a) Time series images of single droplet atomization, probability volume density distribution of fine particles per dimensionless time in single droplet atomization; (b) $\tau=1.0$, (c) $\tau=2.0$ 


\section{Conclusions}

Particles of known size were photographed at arbitrary particle-focus distances to obtain a database of particle size, particle depth position, and particle images. Using the least-squares method, two approximate surface equations, one to identify the depth position $z$ and the other to identify the contour brightness threshold of the particle, were derived from the database. In addition, it was determined that the solution could not be obtained algebraically from the two surface equations and the three particle images, and a method for measuring the depth position and particle size using loops was devised. The volume probability density distribution was obtained using the particle-size measurement method. It is known that the volume probability density distribution of fine droplets in droplet atomization follows the root-normal distribution, and the volume probability density distribution obtained by this method also follows a root-normal distribution.

\section{Acknowledgments}

This work was supported by JSPS KAKENHI Grant Number JP19J13109 and Grant-in-Aid for Research on Atomization and Spray Systems from ILASS-Japan, No. 2020-02.

\section{References}

[1] Eshel, G., Levy, G., Mingelgrin, U., Singer, M., 2004, Soil Science Society of America Journal, 68, pp. 736-743.

[2] Park, S.W., Kim, S., Lee, C.S., 2006, International Journal of Multiphase Flow, 32, pp. 807822.

[3] Yatsufusa, T., Kidoguchi, Y., Adam, A., Gomi, T., 2009, atomization(in Japanese), 18, pp. 8895.

[4] Takagaki, N., Iwano, K., Ilyasov, E., Komori, S., and Shirakawa, Y., Development of an optical imaging technique for particle number density, Journal of Fluid Science and Technology, 13, 2008 , pp. 1-13.

[5] Kamiya, T., 2020, the 29 th ILASS-Japan Symposium (in Japanese).

[6] Asahara, M., Hattori, Y., Miyasaka, T., Kamiya, T., 2020, Transactions of the JSME (in Japanese), 86, pp. 1-12.

[7] Guildenbecher, D.R., Gao, J., Chen, J., Sojka, P.E., 2017, two-view, digital in-line holography. International Journal of Multiphase Flow, 94, pp. 107-122. 